\title{
Sulfonamide Resistance in Chicken Coccidiosis: A Clinico-Pathological Study
}

\author{
AMAM Zonaed Siddiki ${ }^{*}$, M Jahurul Karim ${ }^{1}$ and Emdadul Haque Chawdhury ${ }^{2}$ \\ ${ }^{1}$ Department of Pathology \& Parasitology, Faculty of Veterinary Sciences, Bangladesh Agricultural University (BAU), Mymensingh 2202, \\ Bangladesh, ${ }^{2}$ Department of Pathology, Faculty of Veterinary Sciences, Bangladesh Agricultural University (BAU), Mymensingh 2202, Bangladesh
}

[Received 20 February 2008; Accepted 14 June 2008]

\begin{abstract}
Coccidiosis is a disease of great economic importance for the emerging poultry industry of Bangladesh. Sulfonamides have been used extensively for treatment and control of chicken coccidiosis. However, there have been complaints regarding failure of $\mathrm{Esb}_{3}{ }^{\circledR}$ (sodium sulfachloropyrazine monohydrate; Ciba-Geigy) in treating clinical coccidiosis. Therefore this study was undertaken to examine the degree of sulfonamide resistance of field coccidian isolates. Four groups of 12-day-old chicks were infected with $10^{4}$ sporulated oocysts of four field coccidian isolates. Each group contains four subgroups containing 8 chicks in each subgroup, among which one subgroup remained as infected untreated control and another 3 subgroups were treated with $30 \% \mathrm{Esb}_{3}{ }^{\circledR} @ 1$, 2, or $2.5 \mathrm{~g} / \mathrm{l}$ of drinking water for 3 consecutive days. The sulfonamide resistance was assessed on the basis of faecal oocyst counts and clinico-pathological findings. There was no significant difference $(p>0.05)$ in weight gain between untreated chicks and the chicks treated @ 1 and 2 g/l. However, a significantly higher $(p<0.05)$ weight gains were noted in subgroups treated with $\mathbf{E s b}_{3}{ }^{\circledR} @ 2.5 \mathrm{~g} / \mathrm{l}$. Chicks received treated with $2 \mathrm{~g} / \mathrm{Esb}_{3}{ }^{\circledR}$ received similar clinical coccidiosis and histopathological changes but little less oocyst counts as found in untreated infected control chicks. Chicks treated with $2 \mathrm{~g} / \mathbf{~ E s b}_{3}{ }^{\circledR}{ }^{\text {did }}$ not reveal clinical coccidiosis and revealed no or very minimal oocyst counts during the treatment period. But on histopathology, arrested or stunted parasitic stages were found during the treatment period whereas flourish parasitic development with subacute-chronic ulcerative caecitis was evident following withdrawal of treatment. These findings suggest that the drug only cause an arrested parasitic development rather than killing the parasite. Therefore, an alternative to the sulfonamides needs to find out for treating and controlling chicken coccidiosis.
\end{abstract}

Keywords: Chicken, Coccidiosis, Sulfonamide, Resistance

\section{Introduction}

Coccidiosis is a common parasitic disease of broiler chickens caused by single-celled protozoan parasites of the genus Eimeria, which are commonly referred to as coccidia ${ }^{1}$. There are two types of coccidiosis, clinical coccidiosis in which the affected birds show typical symptoms of the disease, such as bloody droppings and increased mortality. The other type is known as subclinical coccidiosis because the affected birds do not show visible symptoms of the disease but when a random sample of birds is examined, the presence of the gross lesions and the coccidia are found ${ }^{1}$. Coccidiosis is probably the most prevalent infectious disease confronting the poultry industry, and as such it is a protozoan disease of the intestinal tract of chickens and other birds, usually subact or chronic in its course, but sometimes acute in its manifestation. It is most injurious to young chicks, although older fowls are often seriously affected. In rate cases the disease in geese assumes an unusually and highly fatal form in which the kidneys are the organ principally involved ${ }^{1}$.
Coccidiosis has been identified as a major threat in chickens in Bangladesh, which causes a great economic loss to the poultry industry $^{2-3}$. The subclinical form of the infection causes an enormous economic loss particularly through reduced weight gain in broilers. Oral broad-spectrum antibiotic intended for treatment of coccidiosis and specific bacterial diseases in poultry. A number of sulfonamides are available for treatment and prevention of chicken coccidiosis in Bangladesh. Among these, $\mathrm{Esb}_{3}{ }^{\circledR}$ (CibaGeigy), contains 30 g sulfaclozine (sulfachloropyrazine) sodium monohydrate per $100 \mathrm{~g}$, is used extensively throughout the country. Like all sulfonamides, sulfaclozine is a competitive antagonist of para-aminobenzoic acid (PABA), a precursor of folic acid, in protozoa and bacteria. Folic acid is a coenzyme necessary for the synthesis of nucleic acid, hence sensitive species do not multiply in the presence of $\mathrm{Esb}_{3}{ }^{\circledR}$. The manufacturers claimed a standard treatment for 3 consecutive days with $1 \mathrm{~g}$ of $\mathrm{Esb}_{3}{ }^{\circledR}$ per litre of drinking water, which affects the early second generation schizonts of Eimeria sp. can cure clinical coccidiosis in chicken. However, over the years there have been complaints regarding

${ }^{*}$ Corresponding authors:

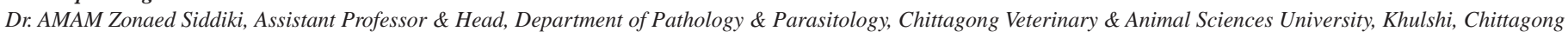
4202, Bangladesh

Tel (Office): (031) 659093; Cell: 01717 718884; Fax: +880 (031) 659620; E-mail: zsiddiki@gmail.com 
failure of $\mathrm{Esb}_{3}{ }^{\circledR}$ in treating clinical coccidiosis in Bangladesh, but there is no published report. Therefore, this study has been designed to examine the degree of sulfonamide resistance of field coccidian isolates.

\section{Materials \& Methods}

\section{Chicks and experimental design}

The experiment was conducted at the Department of Parasitology and Department of Pathology, Bangladesh Agricultural University (BAU), Mymensingh. A total of 4 caecal isolates (Eimeria tenella) were collected from 4 different poultry farms of Dhaka and Narsingdi district, during the period from December 1999 to March 2000. The oocysts were passed in 10-day-old chicks, cleared and sporulated for subsequent use.

Twelve-day-old Starbrow broiler chicks were used. The chicks were randomly divided into 4 groups (Group A, B, C and D) containing 4 subgroups of each. Each subgroup contained 8 chicks and they were reared in separate cages. Each subgroup was infected with separate field isolate of Eimeria tenella. The infected inoculum was introduced directly into the crop of the chick using a plastic dosing tube attached to a $1 \mathrm{ml}$ plastic syringe. All the chicks belonging to groups A, B, C and D were infected with $10^{4}$ sporulated oocysts of field isolates 1, 2, 3 and 4 respectively. One subgroup each from group A, B, C and D were treated with $\mathrm{Esb}_{3}{ }^{\circledR}$ @ $1 \mathrm{~g} / \mathrm{l}$ of drinking water, one with $2 \mathrm{~g} / \mathrm{l}$ and one with $2.5 \mathrm{~g} / \mathrm{l}$; whereas, one subgroup from each group was remained as untreated control. Esb ${ }_{3}{ }^{\circledR}$ was administered after $72 \mathrm{~h}$ of infection and continued for 3 consecutive days. Clinical signs, oocyst output and weight gain were recorded in groups $\mathrm{A}$ and $\mathrm{B}$, whereas one chick from each subgroup of group $\mathrm{C}$ and $\mathrm{D}$ were sacrificed and necropsied regularly from 3 to 8 days post-infection (dpi).

\section{Clinical findings}

The chicks were observed very closely for recording the clinical manifestations. Faecal samples were collected daily and were prepared as follows: specimens were transferred in a container containing $2 \%$ potassium dichromate, homogenized by adding water. One $\mathrm{ml}$ suspension was again diluted in the saturated salt solution to make a desire solution (x10, x50, or x100). A drop of the suspension was withdrawn with the help of an ordinary plastic transfer pipette and the chamber of the McMaster egg counting was filled. After $5 \mathrm{~min}$, the oocysts present in the two chambers were counted under microscope. The oocyst output per bird was calculated by dividing the figure by 0.3 and multiplied by the dilution factor.

\section{Weight gain}

Chicks were weighed individually from the day of infection till the end of the experiment everyday at the same time, and the mean values were calculated.

\section{Necropsy}

The whole intestine including the caeca was removed. Gross lesions were noted and additionally mucosal scrapings were examined microscopically to study the developmental stages of Eimeria tenella. Caecal tissues were collected and preserved in $10 \%$ neutral buffered formalin.

\section{Histopathology}

The formalin fixed tissues were embedded in paraffin by routine methods. Sections were stained with haematoxylene and $\operatorname{eosin}^{4}$. Stained sections were examined for pathological lesions and as well as for coccidial developmental stages. For comparison of histopathological changes, caecal tissues of 8 uninfected untreated control chicks were examined.

\section{Statistical analysis}

Groups mean values of weight gain of the chicks following infection were compared by a student's $t$-test with $\mathrm{N} 1+\mathrm{N} 2-2$ degrees of freedom ${ }^{5}$.

\section{Results}

\section{Clinical signs}

The untreated infected chicks of all age groups (Group A1, B1, C1 and D1) showed clinical coccidiosis with clotted and unclotted blood in faeces from 4 days post-infection (dpi). The signs aggravated on 5 and 6 dpi and the birds became listless and fresh bloody discharge was found in the droppings. On 6 dpi, one bird infected with isolate 1 and two birds with isolate 2 died from Group A1. Chicks received Esb ${ }_{3}{ }^{\circledR} 1 \mathrm{~g} / \mathrm{l}$ (Group A2, B2, C2 and D2) showed similar clinical signs with those of the untreated groups (Group A1, B1, C1 and D1). Chicks received treatment @ 2 g and/ or $2.5 \mathrm{~g}$ of $\mathrm{Esb}_{3}{ }^{\circledR}$ per litre of drinking water (Group A3,4; B3,4; C3,4 and D3,4) showed very minimal or no clinical signs.

\section{Oocyst output}

The untreated chicks passed a large number of oocysts following infection with both the isolates. The chicks treated with different doses of $\mathrm{Esb}_{3}{ }^{\circledR}$ also passed a variable number of oocysts. The mean output is shown in the Table 1 . There was no significant difference $(p>0.05)$ in weight gain between untreated chicks and chicks treated @ $1 \mathrm{~g} / \mathrm{l}$ and $2 \mathrm{~g} / \mathrm{l}$ except that with one isolate the group treated @ $1 \mathrm{~g} / \mathrm{l}$ had a significantly higher $(p<0.05)$ weight gain compared with untreated control. However, significantly higher $(p<0.05)$ weight gain were noted in groups treated @ $2.5 \mathrm{~g} / \mathrm{l}$.

\section{Necropsy}

The caeca of untreated dead birds sacrificed on 5 to 7 dpi were highly swollen with full of clotted blood and showed large number of haemorrhagic and necrotic spots on the mucosal surface. Among the treated subgroups, birds receiving $\mathrm{Esb}_{3}{ }^{\circledR} @ 1 \mathrm{~g} / \mathrm{l}$ showed almost similar lesions with those of untreated groups. Plenty of merozoites were also observed during 4 to $7 \mathrm{dpi}$. All subgroups receiving treatments @ 2 g/l revealed a hyperemic, swollen and thickened caecal wall with oocysts in mucosal scrapings. The lesions were more pronounced from 6 dpi and a moderate number of oocysts were present till the end of the 
Table 1. Group mean weight gain and oocyst output following infection with sporulated oocysts of Eimeria tenella and treated with different doses of $E s b_{3}{ }^{\circledR}$

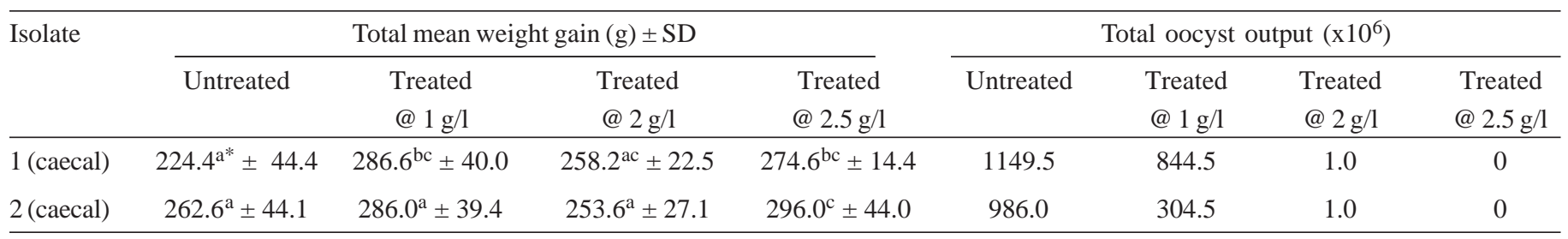

${ }^{*}$ Figures followed by the same superscripts are not significantly different.

experiment. The birds of all subgroups receiving treatment @ $2.5 \mathrm{~g}$ of $\mathrm{Esb}_{3}{ }^{\circledR}$ per litre of drinking water showed hyperemia and mild thickening of caecal wall with presence of small number of oocysts in mucosal scrapings between 5 and $8 \mathrm{dpi}$.

\section{Histopathology}

At 2 dpi slight thickening of the villi was seen due to infiltration of mononuclear cells and eosinophils in lamina propria. Merozoites were found in contact with villi and crypt epithelium. Inflammation proceeded further with additional haemorrhages in the lamina propria and in caecal lumen on $3 \mathrm{dpi}$, which further aggravated at 4 to $5 \mathrm{dpi}$ and developed severe acute haemorrhagic erosive caecitis involving the submucosa. Haemorrhages were extremely severe in lamina propria and in submucosa. Caecal lumen contained desquamated epithelium and bloody exudates. Cells of the villi and crypt contained large number of second generation schizonts (Figure 1A). Additional haemorrhages and inflammation of muscular layer with caecal core formation in the lumen were seen at 6 dpi. Both schizonts and gametes were observed in the crypt and villus epithelium. These lesions were severely aggravated with additional proliferation of immature fibroblast indicating subacute nature of the inflammation on $7 \mathrm{dpi}$ with extreme haemorrhages in submucosa. Almost all epithelial cells and caecal lumen contained gametes and oocysts. Chicks sacrificed on 8 dpi revealed severe subacute to chronic haemorrhagic transmural ulcerative caecitis while remaining non-infected epithelial cells showed hyperplasia. Majority of the villus and crypt epithelial cells and caecal lumen contained gametes and oocysts. Surprisingly, clusters of merozoites were found at centre of one of the lymphatic nodules (Figure 1B).

Chicks infected with E. tenella and treated with $E s b_{3}{ }^{\circledR} @ 1 \mathrm{~g} / \mathrm{l}$ Chicks of all subgroups revealed almost similar histopathological changes with those of untreated subgroups. More severe lesions were seen at 4 dpi where acute diffuse haemorrhagic caecitis was diagnosed. Arrested coccidial development with necrosis and desquamation of epithelial cells was also seen that became extremely severe on 5 and 6 dpi with mononuclear cells (mostly lymphocytes with few macrophages) and eosinophils infiltration and extremely severe haemorrhages in submucosa (Figure 1C) and muscular layer of the caecum. Tiny second generation merozoites were seen at the surface of the crypt epithelial cells. Subacute to chronic transmural caecitis consistent with arrested coccidian oocyst was diagnosed on 7 dpi (Figure 1D). Chicks sacrificed at 8 dpi exhibited more severe necrotizing ulcerative caecitis consistent with plenty of coccidian gametes and oocysts in the epithelial cells and in the caecal lumen.

Chicks infected with E. tenella and treated with Esb ${ }_{3}{ }^{\circledR} @ 2 \mathrm{~g} / \mathrm{l}$ Very minimal or no histopathological changes were found till 5 dpi. Remarkably, bird killed at 6 dpi exhibited severe changes that include acute haemorrhagic caecitis. Haemorrhages were also found in the lymphatic nodules. Coccidian gametes and schizonts were seen in crypt epithelial cells and remaining non-infected epithelial cells showed hyperplastic changes. Following flourish coccidian development the changes become more severe on $7 \mathrm{dpi}$. The inflammatory cells invaded the submucosa and muscular layer with desquamation of epithelial cells. Exceptionally, haemorrhages in muscular layer were extremely severe (Figure 2A). Desquamated epithelial cells with bloody exudates were accumulated in the caecal lumen. Scattered second generation schizonts were watched at the surface of the crypt epithelium. These lesions proceeded further on $8 \mathrm{dpi}$ and a subacute to chronic haemorrhagic ulcerative transmural caecitis was diagnosed. A moderate number of epithelial cells contained coccidian gametes and oocysts where remaining non-infected epithelial cells exhibited hyperplasia.

Chicks infected with E. tenella and treated with Esb ${ }_{3}{ }^{\circledR}$ @ $2.5 \mathrm{~g} / \mathrm{l}$ Very minimal mononuclear cell infiltration with increased goblet cell proliferation was observed till 5 dpi. These lesions proceeded and revealed more severe lesions at 6 dpi that include subacute diffuse erosive caecitis with intralesional coccidia that exhibited stunted growth (Figure 2B). Remarkably severe haemorrhages were found in one of the lymphatic nodules. Lymphatic nodules and non-infected epithelial cells showed extreme hyperplasia. These lesions advanced further and which became chronic transmural caecitis at $8 \mathrm{dpi}$. A small number of intra- and extracellular coccidian gametes and oocysts were seen. 

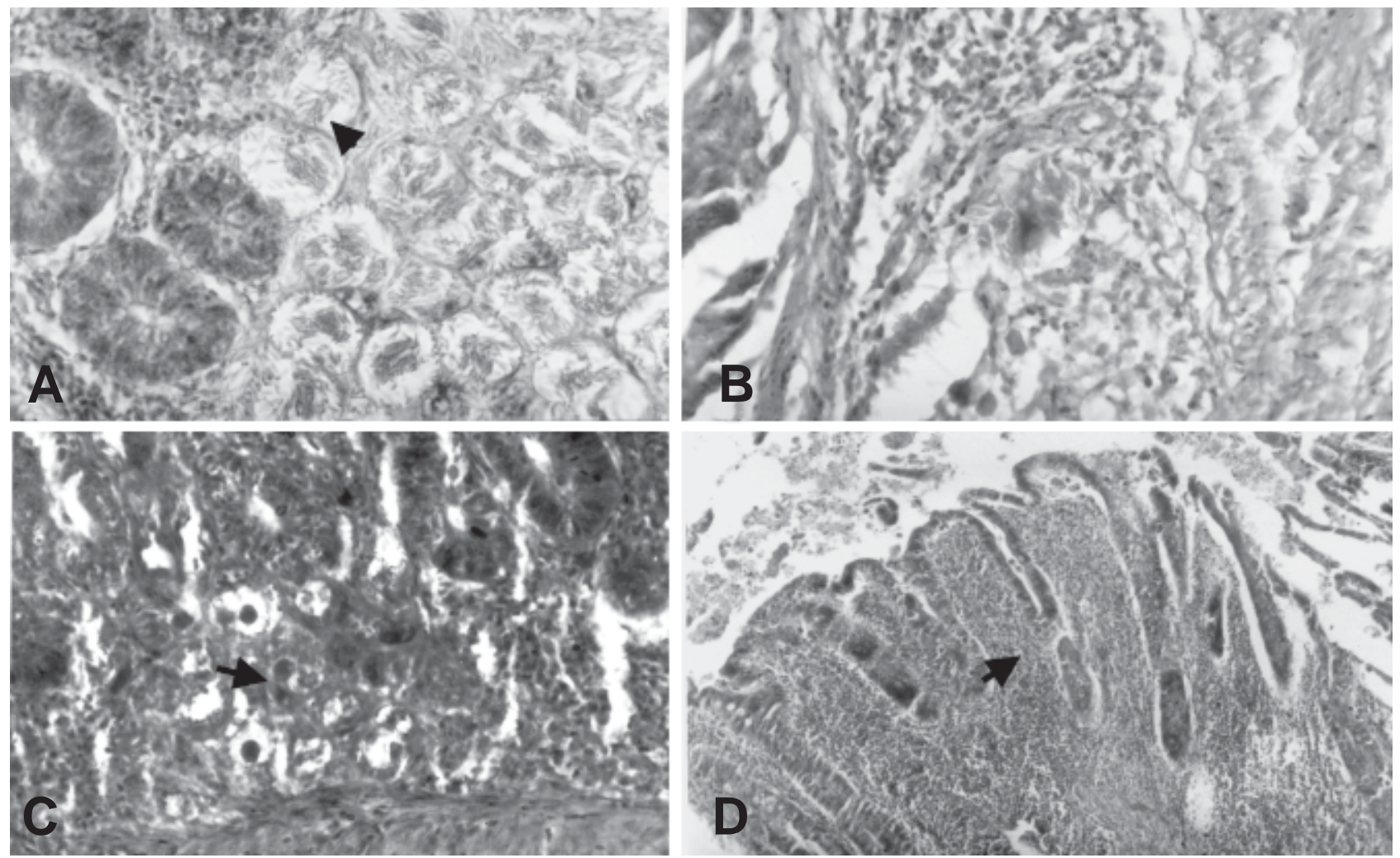

Figure 1. Caecal section of chicks infected with Eimeria tenella, sacrificed at 5 dpi and untreated, shows fully mature second generation schizonts (arrow) containing merozoites (A) ( $H \& E$, x330), sacrificed at 8 dpi and untreated, shows clusters of merozoites in the lymphatic nodule (B) (H \& E, x330), sacrificed at 5 dpi and treated @ $1 \mathrm{~g}$ of Esb ${ }_{3}^{\circledR}$ per litre of drinking water, shows severe haemorrhages in the submucosa with stunted coccidial schizonts (arrow) at the side of the haemorrhages $(C)(H \&$ $E, x 82.5)$ and severe subacute to chronic caecitis consistent with tiny coccidian gametes (arrow) (D) (H \& E, x330).
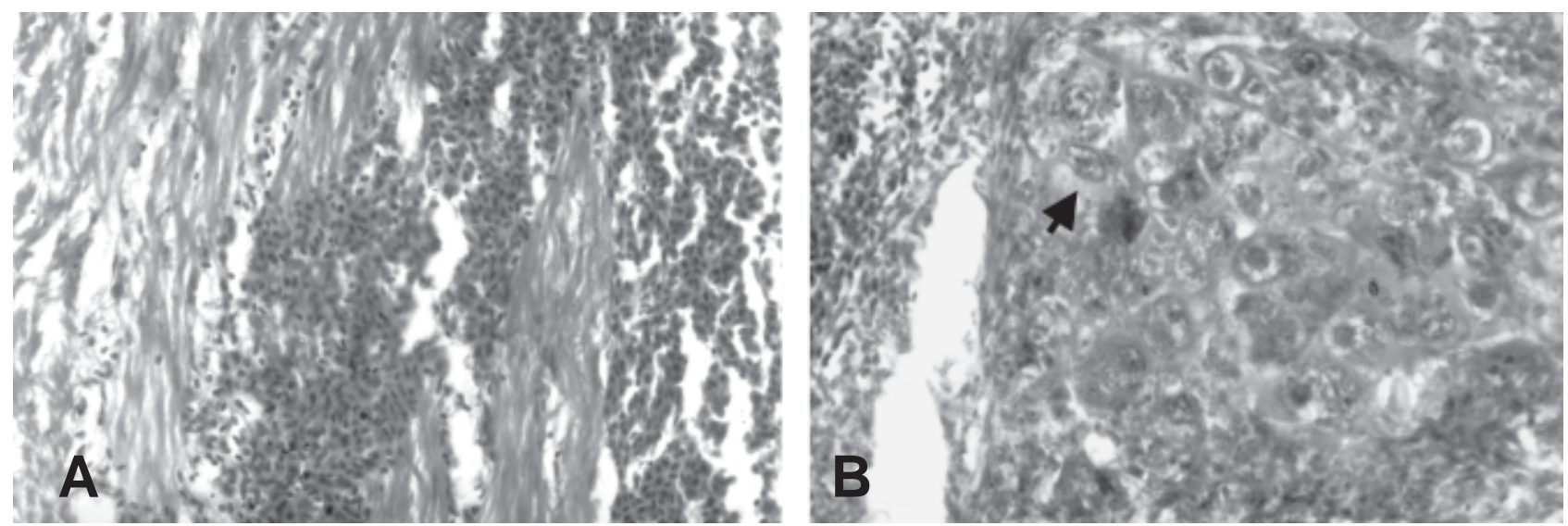

Figure 2. Caecal section of chicks infected with Eimeria tenella, sacrificed at 7 dpi and treated @ $2 \mathrm{~g}$ of Esb ${ }_{3}{ }^{\circledR}$ per litre of drinking water, shows severe haemorrhages in muscular coat of the caecum (A) (H \& E, x330), sacrificed at 6 dpi and treated @ $2.5 \mathrm{~g}$ of $\mathrm{Esb}_{3}{ }^{\circledR}$ per litre of drinking water, shows subacute caecitis consistent with stunted coccidial development (arrow)(B) (H \& E, x330).

\section{Discussion}

A special committee of the World Health Organization (WHO) defines drug resistance in 1963 as the absence or insufficiency of drug action after administration of a normally effective dose. Passing out of relatively large number of oocysts by the chicks treated @ 1 g/l of drinking water, the original recommended dose of $\mathrm{Esb}_{3}{ }^{\circledR}$ is a clear indication that used coccidian isolates had the ability to remain viable and of multiplication. This statement is further supported by the similar histopathological findings both in untreated chicks and chicks treated @ $1 \mathrm{~g} / \mathrm{l}$. This clearly demonstrates a degree of resistance by the coccidia against the said drugs. Demonstration of a high degree of efficacy and complete cessation of oocyst production at 3 days post treatment with $\mathrm{Esb}_{3}{ }^{\circledR} @ 1 \mathrm{~g} / \mathrm{l}$ suggest that in the absence of resistance, $\mathrm{Esb}_{3}{ }^{\circledR}$ is highly effective in treating chicken coccidiosis $^{6-7}$. 
Demonstration of resistance was also substantiated by the fact that immediately after cessation of treatment at 5 days postinfection (dpi) the parasitic stages started developing very quickly, which was authenticated by the presence of a good number second generation schizonts and merozoites. Stunted coccidian growth during the treatment period and transmural caecitis at histopathological examinations from 6 dpi and more and more developmental stages at 7 and 8 dpi further explain the presence of sulfonamide resistance in the Eimeria sp. used in this study. However, demonstration of arrested or stunted coccidian stages at $4 \mathrm{dpi}$, which were more pronounced at $5 \mathrm{dpi}$, suggested that the drug could at least suppress the development of the parasite although could not totally destroy or kill the developing stages. This further indicates that the suppression in development was a temporary phenomenon, which on the withdrawal of the drug was relaxed and allows further development of the parasite. However, relatively fewer oocyst outputs compared with the untreated control group indicate that at least some of the parasitic stages might have been destroyed.

Lack of total cessation of oocyst production in group following treatment @ 2 g/l of $\mathrm{Esb}_{3}{ }^{\circledR}$ (Table 1) clearly suggests that the isolates had developed a significant level of resistance against the drug. At this dose level, the drug was however capable of keeping the pathological lesions at the very minimal level at least during the period of treatment. But severe haemorrhagic caecitis at 6 dpi and a flourish coccidial development with presence of a large number of second generation schizonts and gametes and oocysts in almost all the cells at 8 dpi suggests that even at this dose level the drug was not capable of killing the parasites. However, the suppression in development was little more pronounced that what had seen in chickens receiving $1 \mathrm{~g} / \mathrm{l}$. This means the drug caused a more reduction in the rate of development of the parasitic stages. These clearly suggest a good degree of resistance in most of the isolates used in this study.

Many workers have also reported resistance against sulfonamides from different countries of the world ${ }^{8-13}$. It is therefore very important to note that since the demonstration of the effect of sulfonamide against chicken coccidia by Horton-Smith and Taylor in the year 1942, it took only 12 years for the development of a highly resistant strain of Eimeria ${ }^{9}$. Sulfonamides have been widely and of course indiscriminately used in the treatment of chicken coccidiosis in Bangladesh since early seventies. Therefore, it is very likely that the coccidian species would develop a degree of resistance against these drugs.

The dose rate of sulfonamides is an important factor in preventing or treating the disease. When given in recommended amounts, the sulfonamides are not generally harmful, but higher concentrations are usually toxic ${ }^{14}$. Increasing the concentration of drug may not be feasible, as this may sometimes at a level become toxic to the bird. Therefore, because of the fear of toxicity, it would not be advisable to increase the dosage of $\mathrm{Esb}_{3}{ }^{\circledR}$ to face the challenge of resistant strains. Moreover, one can not ignore the possible danger of residual effect of this drug in the meat and also the high cost of the treatment.

However, although the clinico-pathological findings of coccidiosis are established in few several studies ${ }^{1,15}$, this investigation revealed few additional interesting findings for example, haemorrhage in lymphatic nodules, submucosa and in muscular coat. Schizonts in the lymphatic nodules are surprising. Coccidia are generally epitheliotrophic. Haemorrhages in lymphatic nodules, submucosa and muscular coat probably either due to the toxic products of damaged epithelial cells which damaged the blood vessels or coccidia may infect the blood vessel endothelium. However, coccidial developmental stages were not found in the endothelium. This needs further investigation.

Therefore, since all the isolates used in this study showed a degree of resistance, it is considered to be great concern for the developing poultry industry in Bangladesh. Increasing the dosage of the drug, as suggested by the manufacturers, can not be right answer to face this problem, since this can open another dimension by inflicting toxic effects to the birds. Therefore, an alternative to the sulfonamides needs to find out for treating and controlling chicken coccidiosis.

\section{Acknowledgements}

The authors are grateful to Bangladesh Agricultural University Research System (BAURES) for financial support of this study.

\section{References}

1. McDougald LR \& Reid WM. 1991. Coccidiosis. In Diseases of Poultry (Calnek BW, Johnbarne H, Beard CW \& Yoder HW eds), $9^{\text {th }}$ edn, pp 780-797. Iowa State University Press, Ames, Iowa.

2. Karim MJ \& Trees AJ. 1990. Isolation of five species of Eimeria from chickens in Bangladesh. Trop Anim Health Prod. 22: 153-159.

3. Karim MJ, Begum N \& Khan MSR. 1994. Prevalence of coccidiosis in broilers on two farms in Bangladesh. Pak J Sci Ind Res. 37: 339-341.

4. Luna LG. 1968. Manual of Histologic Staining Methods of the Armed Forces Institute of Pathology, $3^{\text {rd }}$ edn. McGraw Hill Book Company, New York.

5. Bailey NTJ.1981. The use of $t$-test for small samples. In Statistical Methods in Biology, pp 43-51. Hodder \& Stoughton, London.

6. Rahman SA, Sathyanarayan ML \& Placid EDS. 1993. Efficacy of $\mathrm{Esb}_{3}$, a new anticoccidial drug. Indian Vet J. 70: 949-951.

7. Padmavathi P, Ramesh AJ \& Gangadhar Rao YVB. 1995. Studies on the therapeutic efficacy of $\mathrm{Esb}_{3}$ against experimental mixed coccidial infection in chickens. Indian Vet J. 72: 690-693.

8. Cuckler AC \& Malanga CM. 1955. Studies on drug resistance in coccidia. J Parasitol. 41: 302-311.

9. Weletzky E, Neal R \& Hable I. 1954. A field strain of Eimeria tenella resistant to sulfonamides. J Parasitol. 40(Suppl): 24.

10. Warren EW, Ball SJ \& Mackenzie DR. 1966. The incidence of drug resistant strains of Eimeria sp. in chickens in Great Britain. Br Vet J. 122: 534-543.

11. Hodgson JN, Ball SJ, Ryan KC \& Warren EW. 1969. The incidence of drug resistant strains of Eimeria in chickens in Great Britain. Br Vet $\mathrm{J}$. 125: 31-35.

12. Gill BS \& Bajwa RS. 1979. Drug resistance in field isolates of chicken coccidia from Punjab state. Indian J Parasitol. 3: 131-134.

13. Krylov VF \& Zaionts VI. 1981. The prevalence of drug resistant cultures of coccidia. Veterinariya Moscow. 3: 45-46.

14. Levine ND. 1961. Protozoan Parasites of Domestic Animals and of Man, $1^{\text {st }}$ edn. Burgess Publishing Company, Minnesota.

15. Panda DN, Mishra A, Mishra SC \& Mohapatra HK. 1996. Comparative clinicopathological changes between Eimeria tenella infected and $\mathrm{Esb}_{3}$ treated broiler chicks. Indian J Anim Sci. 67: 491492. 